\title{
MAGNETIZATION AND SPIN SUSCEPTIBILITY OF AN INTERACTING TWO-DIMENSIONAL ELECTRON GAS
}

\author{
A. L. Subaşı and B. Tanatar \\ Bilkent University, Department of Physics, Bilkent 06800, Ankara, Turkey
}

\begin{abstract}
We study the magnetic behavior and in particular the spin susceptibility of an interacting two-dimensional electron gas in a finite in-plane magnetic field. The total energy of the system is constructed using the recent quantum Monte Carlo simulation based parametrized correlation energy as a function of density, spin polarization, and applied magnetic field. The critical magnetic field to fully spin polarize the system is obtained as a function of the electron density. The spin polarization as a function of the applied field (less than the critical field) for various densities are calculated. The spin susceptibility as a function of the applied field for various densities are calculated. The zero-field value of the spin susceptibility as a function of electron density is compared with relevant experiments.
\end{abstract}

Keywords: 2D electron gas, Spin polarization, Nonlinear Susceptibility, Many body effects

PACS: $71.10 . \mathrm{Ca}, 71.45 . \mathrm{Gm}$

The spin susceptibility of a two-dimensional electron gas (2DEG) as occurs in GaAs based semiconducting structures has been of great experimental interest[1] recently in connection with the apparent metal-insulator transition observed in such systems. [2] Theoretical calculations of the spin susceptibility mostly relay on accurate ground state energies provided by quantum Monte Carlo (QMC) simulations. $[3,4,5]$ The experiments reveal highly nonlinear behavior which is crucial for making contact with theoretical calculations. Furthermore, experiments are typically performed with an in-plane applied magnetic field which needs to be taken into account in analyzing the spin susceptibility. More recently nonlinear effects in high fields and at low densities have been investigated within the random phase approximation (RPA) $[6,7]$. Our aim in this work is to use the more accurate ground state energies from QMC calculations to investigate the nonlinear spin susceptibility in $2 \mathrm{DEG}$ systems.

We consider a strict 2DEG system at zero temperature, $T=0$, with an applied parallel magnetic field. The 2DEG is conveniently characterized by the following two dimensionless parameters. The Wigner-Seitz radius $r_{s}$ is the radius of the circular area per electron in terms of the effective Bohr radius and is related to the density by $n=1 / \pi\left(a_{B}^{*} r_{s}\right)^{2}$. The interaction parameter $r_{s}$ is proportional to the ratio of the average interaction energy to average kinetic energy, $V / K \sim r_{s}$, and characterizes a more strongly interacting system as it increases. The polarization $\xi$ is the ratio of the number of excess electron spins to the total number of electrons, $\xi=\left|n_{\uparrow}-n_{\downarrow}\right| / n$. In a fully polarized or ferromagnetic state $(\xi=1)$ all spins are aligned and an unpolarized or paramagnetic state $(\xi=0)$ means equal number of spin-up and spin-down electrons.
The total energy per particle $E\left(\boldsymbol{r}_{s}, \boldsymbol{\xi}, B\right)$ takes the form

$$
E=E_{k}\left(r_{s}, \xi\right)+E_{x}\left(r_{s}, \xi\right)+E_{c}\left(r_{s}, \xi\right)+E_{Z}(\xi, B)
$$

where $E_{k}=\left(1+\xi^{2}\right) / r_{s}^{2}$ is the kinetic energy, $E_{x}=$ $4 \sqrt{2} /\left(3 \pi r_{s}\right)\left[(1+\xi)^{3 / 2}+(1-\xi)^{3 / 2}\right]$ is the exchange energy, $E_{C}$ denotes the correlation energy and $E_{Z}=$ $-g \mu_{B} a_{B}^{2} \xi B / e^{2}$ is the Zeeman energy in units of Rydberg.

The energy per particle as a function of polarization for different values of the $B$-field is shown in Fig. 1 for $r_{s}=25$, which shows the form of the energy as a function $\xi$ for the paramagnetic electron gas close to the spontaneous phase transition. At zero magnetic field QMC calculations show that the minimum energy occurs at $\xi=0$ for $r_{s}<r_{s}^{*} \sim 25$, the critical density at $B=0$, so that the ground state is paramagnetic. For $r_{s}>r_{s}^{*}$ the fully polarized state has lower energy and the system undergoes a spontaneous phase transition at $r_{s}^{*}$. [4] At a finite applied magnetic field the energy minimum occurs at nonzero polarization $0<\xi^{*}<1$. As $B$ increases the energy at $\xi=1$ equals its local minimum value at $\xi^{*}$ at the critical field $B_{c}$. Beyond the critical field the energy minimum is at $\xi^{*}=1$ and the system is fully polarized. The transition to the ferromagnetic state near the critical density $r_{s}^{*}$ happens with a discrete jump $\Delta \xi^{*}$ in the polarization (see the inset in Fig.2) indicating a first order transition to fully polarized state.

The critical field $B_{c}$ in units of its noninteracting value at the same density $B_{c 0}=\varepsilon_{F} / \mu_{B}$ as a function of $r_{s}$ is shown in Fig.2. The system above this curve in $B-r_{s}$ plane is fully polarized. Reading the figure in the other direction, this curve gives the critical density for the onset of full spin polarization at a given magnetic field, which is the saturation of the optimum polarization 


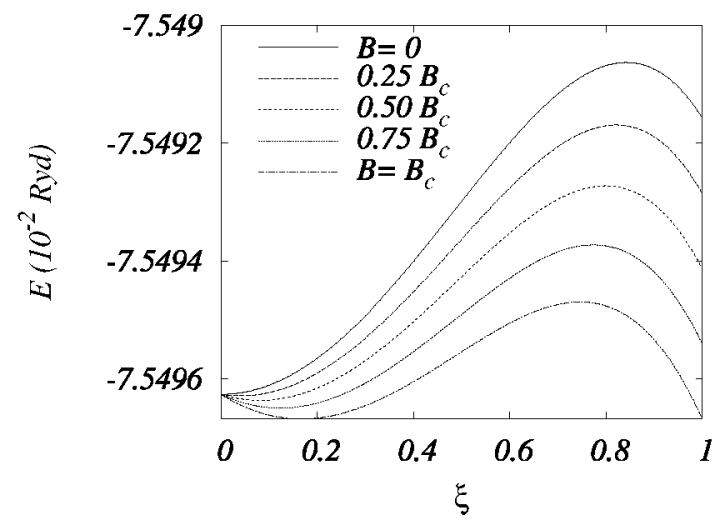

FIGURE 1. Energy as a function of spin polarization at $r_{s}=25$ shown for increasing magnetic field values.

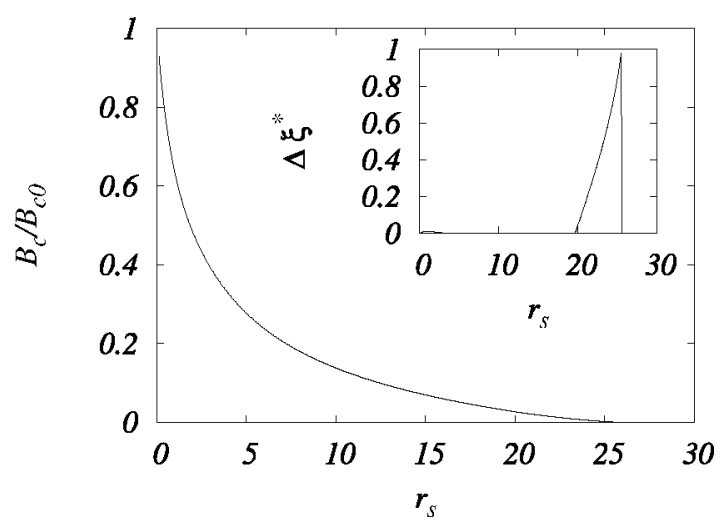

FIGURE 2. The critical field to fully polarize the 2DEG as a function of $r_{s}$. The jump in polarization is shown in the inset.

$\xi^{*}\left(r_{s}\right)$, corresponding to the magnetization of the system, at 1 as the density is decreased at constant $B$.

Our results are in qualitative agreement with those of Zhang and Das Sarma [6] and should be more accurate because we use the more sophisticated QMC energy expression which takes the correlation effects into account fully. The critical $r_{s}$-value is around $r_{s}^{*} \sim 25.5$ which is larger than $r_{s}^{*}=5.5$ of the RPA based calculations. [6, 4]

The polarization $\xi^{*}$ as a function of the magnetic field $B$ at various $r_{s}$ values is shown in Fig.3. The nonlinear behavior being purely an interaction effect is already evident looking at the magnetization curves $\xi^{*}(B)$. From the derivative of $\xi^{*}$ vs. $B / B_{c 0}$ curves the susceptibility can be obtained as a function of the magnetic field.

Different regimes of the spin susceptibility $\chi$ and relevance to experiments is discussed in [6]. The tilted field measurements leading to another quantity, referred to as the semilinear spin susceptibility $\chi_{S}^{*}$ in [6], is found to be reasonable. It coincides with the linear zero field susceptibility at $B=0$. Fig. 4 shows the zero field linear susceptibility as a function of $r_{s}$.

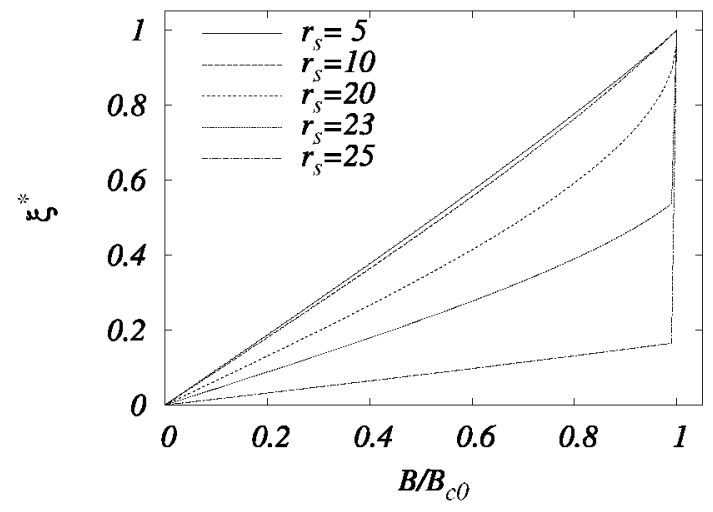

FIGURE 3. Spin polarization $\xi^{*}$ as a function of the $B$-field for different $r_{s}$ values with high nonlinearity at high $r_{s}$ and $B$.

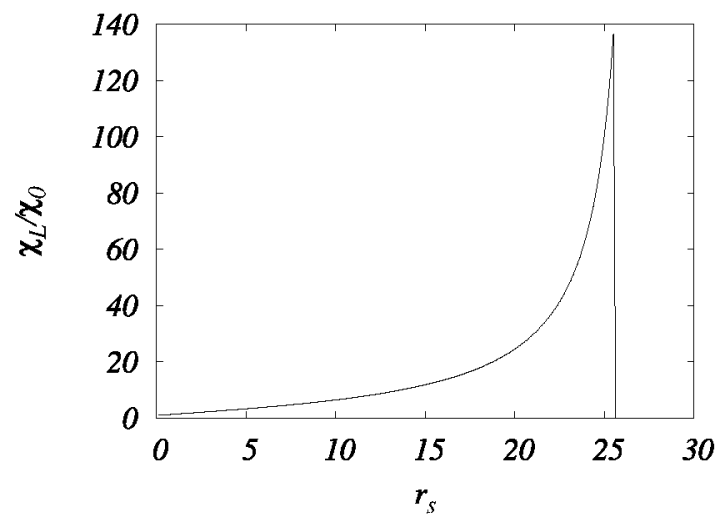

FIGURE 4. Linear susceptibility normalized by the noninteracting Pauli susceptibility at the same density $\chi_{L} / \chi_{0}$ as a function of $r_{S}$ shows enhancement near the critical density.

In summary, we have calculated the spin susceptibility of an interacting $2 \mathrm{DEG}$ using the accurate ground state energies provided by QMC simulations. We have determined the critical value of the applied in-plane magnetic field to fully spin polarize the system.

\section{REFERENCES}

1. E. Tutuc et al., Phys. Rev. Lett. 88, 36805 (2002); J. Zhu et al., Phys. Rev. Lett. 90, 56805 (2003); K. Vakili et al., Phys. Rev. Lett. 92, 226401 (2004); Y.W. Tan et al., Phys. Rev. B 73, 045334 (2006); M. Kumar et al., Solid State Commun. 135, 57 (2005).

2. E. Abrahams et al., Rev. Mod. Phys. 73, 251 (2001).

3. B. Tanatar and D.M. Ceperly, Phys. Rev. B 39, 5005 (1989).

4. C. Attaccalite et al., Phys. Rev. Lett. 88, 256601 (2002).

5. S. De Palo et al., Phys. Rev. Lett. 94, 226405 (2005).

6. Y. Zhang and S. Das Sarma, Phys. Rev. Lett. 96, 196602 (2006).

7. J. Terada and T. Ando, Physica E (in press) 